\title{
L'expertise à l'œuvre : le cas de la coopération européenne en Bolivie
}

Anne Le Naëlou

\section{OpenEdition}

\section{Journals}

Édition électronique

URL : http://journals.openedition.org/apad/1853

DOI : 10.4000/apad.1853

ISSN : 1950-6929

Éditeur

LIT Verlag

Édition imprimée

Date de publication : 1 décembre 1994

Référence électronique

Anne Le Naëlou, «L'expertise à l'œuvre : le cas de la coopération européenne en Bolivie », Bulletin de I'APAD [En ligne], 8 | 1994, mis en ligne le 22 novembre 2007, consulté le 08 septembre 2020. URL : http://journals.openedition.org/apad/1853 ; DOI : https://doi.org/10.4000/apad.1853

Ce document a été généré automatiquement le 8 septembre 2020

Bulletin de I'APAD 


\title{
L'expertise à l'œuvre : le cas de la coopération européenne en Bolivie
}

\author{
Anne Le Naëlou
}

1 L'expertise est devenue aujourd'hui une médiation obligée en matière de décision publique de coopération avec les pays du Sud, tant au niveau de la Commission à Bruxelles qu'au niveau de la réalisation locales des aides. "Dotée du monopole légitime d'une compétence attestée fondée sur la spécialisation du savoir et la délégation d'autorité légale" ${ }^{1}$, l'expertise est un mécanisme essentiel de la contractualisation des aides. Etape obligée et constitutive du processus de coopération Nord/Sud, l'expertise contribue à définir officiellement "un sens" à cette intervention en lui conférant la légitimité du savoir.

2 S'interroger sur l'expertise dans le domaine de l'aide trouve son origine d'un constat en forme de paradoxe: les observateurs s'accordent à reconnaître une inflation d'expertise dans le cadre de la coopération internationale, la politique de coopération de l'Europe communautaire n'échappant pas à cette tendance générale, tandis que d'autre dénoncent un manque de préparation, de réflexion et de connaissance préalable aux interventions de développement. Certains enfin, n'hésitent pas à attribuer une part de responsabilité de l'échec des aides à l'omniprésence de l'expertise.

3 Trop ou pas assez d'expertise? Celle-ci ne jouerait-elle pas plutôt le rôle d'un bouc émissaire bien commode et dans ce cas pourquoi ? Au delà de ces prises de positions, il convient de s'interroger sur le rôle exact de l'expertise dans les processus de coopération.

4 Le recours à l'expertise est en quelque sorte inversement proportionnel au "désenchantement" de la coopération internationale, à une prise de conscience de la faillite de l'aide et d'une situation dont la solution ne peut plus être trouvée avec les schèmes de compréhension et les modes d'action traditionnels. Confrontée aux critiques de leurs politiques d'aide, les institutions internationales et les Etats du Tiers monde tendent à déléguer à des spécialistes la charge de trouver les voies et les moyens de relancer la dynamique de l'aide. 
5 Ainsi, alors qu'au début des années quatre vingt la Politique Communautaire de Développement (P.C.D.) s'ouvre à de nouvelles aires de coopération (Asie et Amérique Latine) avec des moyens financiers élargis et de nouveaux instruments d'aide ${ }^{2}$, la Communauté Economique Européenne a un recours accru à l'expertise. Couramment invoquée, contestée, l'expertise n'est pas un prolongement administratif ou un simple transfert de techniques; elle fonctionne comme un prisme à l'articulation d'au moins deux sources d'attentes : celle des décideurs de l'aide et celles des populations locales attributaires. A la conjonction de ces intérêts contradictoires, l'expertise obéit également à une logique propre.

6 Il convient tout d'abord de préciser les contours d'une notion encore très floue (I). Cette conceptualisation préalable est d'autant plus nécessaire que l'expertise, à l'œuvre, ne se laisse pas appréhender facilement De plus en plus proche du pouvoir de décision, ses finalités sont intimement liées à celles des institutions qui y ont recours. Pour les décideurs, il s'agit d'un sujet pointilleux chargé de susceptibilités. Cette approche théorique de l'expertise est ensuite mise à l'épreuve des faits. L'exercice d'expertise ou le travail de l'expert sur le terrain (II), tel qu'il a été observé au cours de la mise en œuvre d'un Programme de Micro Projets Ruraux réalisé dans le cadre de l'aide financière et technique de la C.E.E. en Bolivie ${ }^{3}$ est plus qu'une simple intervention technique. Les experts réalisent un immense travail social et de mise en liaison entre les objectifs de l'institution commanditaire du Programme et les attentes des communautés rurales de l'Altiplano bolivien.

Définition et logique de l'expertise

7 Compte tenu de l'ampleur du phénomène, les recherches sur l'expertise sont rares, et l'expertise reste encore aujourd'hui, en sociologie, mal définie. Dès les années soixante, J. Berque, posait l'hypothèse d'enjeux considérables liés à ce nouvel intermédiaire dans la coopération internationale ${ }^{4}$.

8 A l'origine, ponctuelle et spécialisée, l'expertise a longtemps eu pour objet d'arbitrer des situations conflictuelles dues à des écarts de normes et de règles. Elle peut encore être aujourd'hui, par exemple, comptable, judiciaire ou agricole ${ }^{5}$. Toutefois, depuis une dizaine d'années, dans le domaine de la coopération et du développement, les expertises sectorielles, menant chacune des actions parallèles et parcellaires, tendent de plus en plus à se constituer en un système unifié. Elles se transforment en une expertise internationale intervenant globalement et indistinctement entre la conception, le financement et l'attribution de l'aide.

9 L'expertise internationale partie prenante des processus de transferts d'innovation, se définit comme un mode de communication entre le système culturel des sociétés industrielles et celui des sociétés préindustrielles. Une aliénation croissante de tous les aspects de la vie quotidienne par la technologie et la science, accrue par une formation insuffisante, entrave le processus de socialisation ${ }^{6}$. L'expertise se voit en quelque sorte chargée de réconcilier cette rupture en garantissant une communication entre des compétences de plus en plus de complexes et les acteurs locaux. Déceler les aspirations des populations locales, comprendre les problèmes à résoudre et les rendre intelligibles pour les intégrer à la logique des institutions publiques ou privées, afin de garantir une cohérence minimale des politiques d'aide au développement, est une des tâches qui incombe à l'expertise. La mise en forme de la communication entre une réalité locale et des institutions passe par une codification des demandes locales en termes de besoins. Le primat de l'idée de besoins est au cœur de la démarche de l'expertise; elle lui 
permet de légitimer son intervention pour un changement social. L'idée de besoins conduit à la représentation d'un social parcellaire et atomisé qui tend à effacer les rapports sociaux pour les remplacer par des comportements individuels. Dans cette logique, à des espaces et des situations définis comme singuliers, correspondent des acteurs singuliers caractérisés par des besoins localisés et spécifiques. A l'évaluation de besoins succède une liste d'actions à entreprendre, sur laquelle l'expertise justifie son intervention. Dresser, en effet, une liste de besoins fondamentaux oblige d'établir une liste correspondante de biens et de services eux aussi considérés comme indispensables. Une fonction latente de l'expertise revient alors à diffuser rationnellement des normes importées.

Dans les politiques d'aide au développement, les actions ont pour objectif d'intervenir, soit pour empêcher certaines situations de se reproduire (inondations, famines), soit pour faire apparaitre des états nouveaux (hausse de la production alimentaire, augmentation des troupeaux, vaccination des individus). Le pourcentage, la moyenne, le minimum et le maximum sont autant de mesures qui font du langage statistique la technique d'évaluation privilégiée utilisée par l'expertise. A travers la combinaison de ces chiffres, des réalités diverses sont retraduites et reconstruites afin d'élaborer une représentation de la réalité. La religion du chiffre est le garant et le symbole de la compétence : il n'y a pas d'expertise sans chiffre. L'expertise contribue à la réduction de tout et de tous à "l'existence statistique" et à la technicisation du discours ${ }^{7}$. Acte éminemment politique, le travail de mise en chiffres de l'expertise entraîne de nouvelles différenciations. La traduction des effets d'une conjoncture climatique défavorable sur l'activité d'une région, en taux d'impact de sécheresse ou d'inondations, contribue à créer des zones d'urgences et une hiérarchisation des faits qui légitiment de nouvelles priorités d'action.

11 Traduction statistique et trahison du réel, telle est l'ambiguïté fondamentale dans laquelle s'exerce l'expertise en tant que médiation.

12 Enfin, agissant pour le compte d'une instance de pouvoir, l'expertise est mandatée pour exercer elle-même un pouvoir, soit d'étude et d'évaluation d'un contexte, soit pour préparer un financement; elle ne s'impose pas seule. Sa légitimité lui est conférée de l'extérieur par une autorité qui la sollicite. A ce rapport de dépendance se superpose une relative autonomie à l'égard des institutions. La qualité de son intervention et, éventuellement, son renouvellement dans le temps est déterminée par une multiplicité de juges. Ces juges sont au moins au nombre de trois : l'institution commanditaire de l'expertise, les pairs et, enfin, les populations locales auprès desquelles elle intervient et dont elle attend également une légitimité et une reconnaissance de son travail. A l'articulation de ces jugements, l'expertise dispose d'une marge d'autonomie qui lui confère un pouvoir pluridimensionnel.

13 Forte d'un savoir technique de pointe, intervenant dans une situation conflictuelle, l'expertise est momentanément investie du pouvoir de trancher au nom de la "vérité" objective et à prendre position dans les débats politiques qui sont présentés comme des questions technico-scientifiques ${ }^{8}$. Pour réaliser une étude ou un diagnostic, des données sont rassemblées et des choix sont proposés. Dès lors, un pouvoir réel est exercé par l'expertise : qui dit pouvoir de trancher, dit aussi pouvoir de décider.

14 L'examen des conditions d'acquisition d'une objectivité et d'une scientificité de l'expert 9 , fait cependant apparaître que le travail d'un spécialiste, d'un technicien ou d'un agronome ne devient expertise qu'à certaines conditions. Sans doute peut-on même 
dire qu'il n'y a pas d'expert sans circonstances d'expertise. On conviendra dans cette perspective, que peu importent les différences entre le savant pur, le chercheur appliqué et le technicien. Chacun peut, dans un contexte donné, être défini comme expert. "Le travail intellectuel auquel il se livre ne devient expertise que dans les conditions concrètes d'exercice mettant enjeu un système de rapports socio-institutionnels" ${ }^{10}$. L'expertise est une situation codifiée où s'expérimente un savoir en quête de reconnaissance sociale tout autant que scientifique. L'avis, le conseil, la consultation, l'intervention, le diagnostic sont autant de "situations d'expertises", c'est-à-dire de modèles d'application d'une connaissance ${ }^{11}$. Les situations de crise ou de déstabilisation, les moments conflictuels, les transferts de techniques et d'une nouvelle rationalité, sont des contextes favorables à l'exercice de l'expertise.

En retour, la situation d'expertise fait l'expert ${ }^{12}$. L'exercice de sa compétence et de son autorité vont de soi puisque sa légitimité lui est accordée par la situation. Cette perspective est centrale car, si la situation d'expertise crée l'expert, dès lors, il ne faut pas sous-estimer la stratégie poursuivie par les experts eux-mêmes, fortement intéressés à la production de situations d'expertise. Il y va de leur autorité individuelle et de la constitution d'une identité collective par la reconnaissance de la compétence. L'attitude des experts face aux situations d'expertise n'est donc pas sans effet sur son bon fonctionnement et sa perpétuation dans l'avenir ${ }^{13}$. Procédures complexes et rites de présentation sont des éléments du "système de conditions" qui confèrent une autorité au discours autorisé ${ }^{14}$.

En résumé, instance de médiation, l'expertise opère un permanent travail de traduction des demandes locales pour créer une situation d'intervention. Ce travail de traduction passe par une interprétation statistique, une formulation des demandes en besoins, leur inscription dans une logique du provisoire et une spatialité intégratrice. Articulés dans les termes d'une idéologie de l'urgence, l'ensemble de ces éléments contribuent à mettre en place les conditions d'existence, bref de légitimation des actions politique de l'institution qui la sollicite. Simultanément, l'expertise se définit par une recherche de légitimité sociale et professionnelle. Car, l'expertise n'est pas seulement une idée. Elle s'incarne à travers des hommes qui travaillent sur le terrain. L'analyse des pratiques des experts dans la mise en place et la réalisation d'un projet de développement permet de mieux connaître le profil sociologique de ces médiateurs.

Une profession en action : l'expert sur le terrain

17 Comme toute forme d'action collective, l'implantation et la mise en œuvre d'un Programme de développement reposent sur un minimum d'intégration des comportements des différents acteurs concernés qui poursuivent chacun des objectifs divergents, voire contradictoires. C'est à la faveur d'une conjoncture climatique particulièrement grave (alternance de périodes de sécheresse et d'inondation entry 1982 et 1983) que redémarre le processus de coopération européenne en Bolivie ${ }^{15}$. L'Etat bolivien, l'Europe ${ }^{16}$ et les communautés rurales de l'Altiplano sont ainsi conduits à trouver un consensus momentané sur fond d'urgence. Une telle négociation ne s'instaure pas naturellement, car le "marchandage" y tient un rôle de tout premier plan. $\mathrm{n}$ doit être sans cesse consolidé et passe par un compromis permanent entre des priorités que chaque groupe social cherche à imposer. C'est précisément au moment où les rationalités doivent se conjuguer et les finalités s'accorder, au moment même de la solution et de la décision, donc de la réalisation, qu'intervient l'expert ${ }^{17}$.

Des inventeurs 

Programme reste imprécis ${ }^{18}$. L'absence d'une méthode de travail clairement préétablie, l'incohérence, la dispersion, la non-efficacité, l'éparpillement sont autant de situations qui ne conviennent pas aux experts et les rendent mal à l'aise ${ }^{19}$.

Réinterprétant et se réappropriant, les volontés européennes, le Modèle Opérationnel Type (M.O.T.) est défini en ces termes "le M.O.T. regroupe les micro-projets et actions pouvant être réalisés au niveau des communautés dans le cadre du Programme et une sélection de zones d'intervention. Le M.O.T. préconise une intervention simultanée sur l'eau, les sols et les voies de communication d'un canton ou d'une communauté. L'ensemble des micro-projets du Programme doit se traduire par des actions cohérentes au niveau des communautés, cantons et provinces et ne doit pas conduire à un éparpillement de réalisations ponctuelles peu efficaces et difficiles à contrôler" ${ }^{20}$.

Dans le même temps, les experts s'exercent également à définir leur propre rôle en tant qu'assistance technique en ces termes "Elle doit assurer la coopération des cantons ou des communautés entre eux, former les paysans à une meilleure technicité, garantir l'emploi des réalisations du projet et assurer une intégration du Programme en milieu paysan. En amont des réalisations, elle prépare des responsables communautaires et informe la population, elle assiste à l'élaboration des dossiers de demande et conseille la gestion de l'exploitation. Pendant les réalisations, elle garantit la coopération entre les responsables du Programme et la population, assure un rôle de conseil au plan concret de l'exécution des tâches par les paysans dans la conduite de leurs exploitations. En aval des réalisations, elle permet l'utilisation optimale des micro-projets par une formation sur le terrain des paysans et par une assistance auprès des responsables de canton ou de communauté" 21 .

21 Plus qu'un simple résumé des objectifs du Programme, l'invention de ce cadre d'action par les experts répond à d'autres finalités plus masquées. Mus par une volonté "d'exorciser" les - incertitudes, les experts systématisent l'intervention européenne pour en faire ressortir les termes d'un modèle de développement, et, ce faisant, tentent d'ériger leurs actions en une intervention reconnue scientifiquement. A travers l'élaboration de ce modèle d'action transparaît une volonté de reconnaissance du travail de l'expert: il lui revient, en effet, de savoir choisir la combinaison d'interventions la plus adéquate à chaque situation particulière. Objectifs du Programme et intérêts des experts se trouvent ainsi, pour un temps, confondus.

Des arpenteurs

22 Dès le lendemain de son arrivée, l'expert travaille à délimiter un territoire ${ }^{22}$. Combinant les impératifs, les contraintes et les opportunités politiques locales, il s'attache à délimiter les sous-zones d'intervention du Programme. Un ensemble de critères d'ordre politique, social et technique guide l'expert dans une logique d'exclusion successive.

Une première série de contraintes est d'ordre technique. Les espaces retenus doivent être "dotés du meilleur potentiel", autrement dit être représentatifs d'une concentration satisfaisante de ressources naturelles. A travers les cartes et les plans d'aménagement, l'espace rural des départements est mis à plat et progressivement reconstruit en un territoire évalué, quadrillé et coté. Une aridité excessive, une zone désertique, un désert de sel, des érosions trop fortes, sont autant de raisons qui écartent plusieurs régions du choix final. 

sillonne les communautés dans lesquelles se construit un ouvrage ou pour vérifier la pertinence d'une demande, et atteste par sa présence de la continuité du Programme. Assisté de son équipe, il parcourt sans cesse son espace d'intervention qui s'étend sur plusieurs cantons et tisse un réseau, avec des trajets régulièrement parcourus et des points d'arrêts. Cette toile d'araignée est en partie permanente et en partie retissée au gré des différentes activités, des différentes actions et des différentes époques de l'année.

Ces critères techniques s'accompagnent de critères d'ordre social. L'intervention du Programme doit se faire en priorité auprès de zones de forte densité rurale et où la sécheresse a été la plus durement ressentie. Cette sélection est effectuée sur la base de pourcentages de taux d'impact de sécheresse déjà calculés par les services techniques des "Corporations" dans le cadre du plan d'urgence national Bolivien. Là encore, un certain nombre de régions se trouvent exclues.

suppose des concessions. Un marchandage indispensable pour garantir la concrétisation du Programme est réalisé par les experts. La prise en compte d'intérêts locaux ou d'une préoccupation gouvernementale de réduction des migrations inter-andines sont autant de nouveaux critères que l'expert doit savoir intégrer dans les termes du programme. Enfin, les experts ajoutent un plus. Ils effectueront plusieurs missions dans différentes régions du pays pour se laisser convaincre et émouvoir.

Progressivement, telle une peau de chagrin, les départements se réduisent à des zones d'interventions, sortes d'enclaves d'action du Programme à l'intérieur du territoire national. Deux sous zones sont retenues dans le département de Potosi : Otavi et Uncia et quatre dans le département de Oruro ${ }^{23}$. Dans ces sous-zones, les experts organisent l'implantation des bureaux et leur lieu de résidence, avec ou sans leur famille.

L'expert entreprend tout d'abord de répertorier les protagonistes potentiels des sous-zones, afin de situer les liens officiels et officieux dans la définition des stratégies locales et de caractériser les attentes locales pour créer les conditions d'une mobilisation autour du Programme. L'expert rencontre et fait se rencontrer successivement des représentants d'institutions gouvernementales, des ministères du plan, du commerce extérieur, de l'industrie et du tourisme, des relations extérieures, de la direction de la Corporation régionale de Développement de Potosi et d'institutions non gouvernementales. A Potosi, il établit des contacts avec les représentants des institutions régionales et locales à caractère technique, sociale, et politique ${ }^{24}$.

L'expert identifie également les acteurs internationaux dans la région, détermine leurs zones d'interventions et la nature de leurs actions. Cette identification et cette information sont délicates à obtenir. Elles sont difficilement accessibles à travers les réseaux administratifs nationaux et internationaux officiels et il ne suffit pas non plus de demander aux populations locales. Les communautés rurales n'explicitent pas forcément leur stratégie d'utilisation des différentes aides.

Mobile, l'expert couvre son terrain d'intervention. Dans sa "Land Rover" rouge, il Véritable arpenteur, l'expert délimite un territoire et catalyse des rencontres entre des acteurs qui jusqu'alors s'ignoraient. Elément exogène à la société bolivienne, il répertorie une multiplicité de volontés institutionnelles, nationales et locales, rencontre les acteurs, les administrations et les populations rurales attributaires, assiste à des réunions techniques des politiques. L'expert construit le Programme en 
regroupant une multitude d'initiatives éparpillées, ponctuelles, localisées et sectorialisées dans des institutions qui, en temps normal, ne communiquent pas entre-elles. Il transforme progressivement ces initiatives en actions du Programme, les acteurs individuels et collectifs en attributaires et partenaires de l'aide. Ce faisant, l'expert construit les termes d'une intégration, fil conducteur du Programme.

Des interprètes

31 Dans la sous-zone d'Uncia, au nord du département de Potosi, l'expert européen est un Français. Agé d'une quarantaine d'années, il a laissé dans un premier temps sa famille en région parisienne. Technicien en horticulture, il ne connaît pas l'Amérique latine. Fort de dix ans d'expérience professionnelle, il a travaillé auparavant dans le nord du Mali pour forer des puits dans le cadre de la coopération internationale suisse. Il s'installe dans une petite maison à la sortie du bourg d'Uncia. Une équipe de six ingénieurs boliviens détachés des services de la Corporation de Potosi réside également dans plusieurs maisons voisines. Parmi eux, deux sont spécialisés en infrastructures hydrauliques et les autres en agronomie. Non loin de là, logent également une secrétaire et une assistante sociale. Outre l'organisation du temps et du travail de cette équipe, l'expert se charge de recruter sur place entre quinze et vingt agents de terrain vulgarisateurs qui seront amenés à vivre de façon itinérante dans les communautés attributaires des actions du Programme.

L'organisation quotidienne du fonctionnement du Programme dans les sous-zones se décompose en une multiplicité de tâches. Dès l'ouverture des bureaux des sous-zones, parvient un grand nombre de demandes écrites de la part des communautés paysannes qui veulent bénéficier du Programme. L'expert sélectionne, vérifie et donne un avis personnel. De l'examen de ces demandes ressort une première sélection de requêtes et un calendrier de visites aux communautés retenues pour réaliser un ouvrage technique.

Les visites préalables à la décision de construction d'un ouvrage permettent à l'expert de contrôler la provenance des lettres de demandes, de juger de leur pertinence technique et économique et éventuellement d'identifier d'autres sites favorables à l'implantation de techniques sur les terres de la communauté rurale ${ }^{25}$. Chaque demande est ensuite discutée et négociée (emplacement, dimension, calendrier de travaux de réalisation) avec les autorités des communautés concernées.

Souvent, l'expert et son équipe réorientent les demandes initiales des communautés rurales vers d'autres demandes plus adaptées au Programme. Le travail de normalisation des demandes est une des dimensions essentielles du travail de l'expert sur le terrain. Il tente ainsi d'éviter une demande sociale à laquelle l'intervention européenne est impuissante à répondre. Enfin, les arguments évoqués par les communautés, les impressions provoquées par les demandes, les sentiments ressentis lors des visites sur le terrain, constituent des critères d'ordre subjectifs qui infléchissent largement le processus de sélection final.

Pris quotidiennement dans une logique d'injonctions contradictoires entre rationalité européenne et demandes des communautés, l'expert va à l'essentiel; il est celui qui "va vers" plutôt que celui qui "accueille". Il bouscule le temps, va de l'avant, n'a pas le temps d'attendre. Lors des parcours avec ses techniciens boliviens ou les membres d'une communauté rurale, l'expert règle plusieurs questions de travail et décide ce qu'il va dire ou faire à son arrivée dans la prochaine communauté visitée. Là aussi, il va bouleverser les habitudes et les rythmes quotidiens en organisant spontanément des 
réunions. Sa personnalité et son charisme s'avèrent, dans ces moments, déterminants pour la qualité et l'intensité des contacts établis. En général, lors de ces réunions, l'expert est à la fois organisateur, animateur et rapporteur. Il se débrouille, sans réelle formation en la matière, en fonction de ses ressources personnelles, de sa maîtrise des situations inédites, de sa capacité à imposer sa logique lors des réunions de contacts avec une assemblée communautaire ou pendant les séances de travail avec son équipe technique ou lors des prises de décision. Dans les réunions s'établit un rapport de pouvoir en faveur de l'expert. Souvent seul à posséder les informations importantes, il est aussi le seul à connaître le but à atteindre et dès lors sa parole vaut "double" Conciliateur, l'expert se fait alternativement porte-parole du Programme auprès des communautés rurales et porte-parole des communautés rurales auprès du Programme. Cette double étiquette lui donne un pouvoir de manipulation: le Programme, et à travers lui, les autorités de Bruxelles et les communautés rurales doivent d'une certaine façon se fier aux experts, seuls points de contacts entre eux.

Des "hommes orchestres"

L'expert est, d'une certaine façon, "taillable et corvéable" à merci. L'observation du Programme entre 1983 et 1986 montre, par exemple, que les fondateurs du Programme auront tous été appelés à se remplacer mutuellement à Potosi, à Otavi ou à Uncia avant d'être remplacés par d'autres experts appartenant aux mêmes bureaux d'études. Cette mobilité des hommes dans les différentes fonctions du Programme révèle une logique implacable. L'homme tend à être dissocié de ses activités de façon à ce que l'activité reste, même quand change son auteur. En pratique, les réactions sont très mitigées. Si ces changements sont relativement peu remarqués par les administrations boliviennes et européennes, ils donnent lieu, au contraire, à de vives réticences de la part des experts et de la part des communautés rurales. L'expert a en général choisi son poste en valorisant soit un travail administratif et politique à Potosi auprès de la "Corporation", soit un travail de terrain avec les communautés rurales dans une sous-zone reculée du département. Pour les communautés rurales, le départ d'un expert est ressenti comme la fin d'une relation sociale de respect et d'amitié qui a parfois pu s'établir en quelques mois de travail commun. On voit toute la différence entre le titre d'expert théoriquement défini à Bruxelles, et le poste réellement occupé 26. Ces hommes sont d'une certaine façon investis d'une double autorité qui se contrarie ${ }^{27}$. Le travail de l'expert sur le terrain qui "sent les choses" vient en quelque sorte modifier et se confronter au titre technocratique initialement défini, d'un commun accord, entre l'expert, son bureau d'étude et Bruxelles.

Susceptible de changer, à tout moment de fonctions, à l'intérieur du cadre administratif du Programme, l'expert change également de fonctions plusieurs fois par jour. Il se fait, tour à tour, plombier et cuisinier chez lui, formateur, électricien et professeur de règle de trois au bureau, maçon avec son équipe dans la maison d'un ami, négociateur avec les autorités de la ville d'Uncia, de la mine ou des communautés rurales, diplomate avec les représentants du gouvernement, expert pour accueillir les missions de Bruxelles.

L'expert est également sur les chantiers. Puits, réservoirs d'eau, bains de déparasitage pour les troupeaux, serres, réseaux d'irrigation, petits ponts, chemins et routes, expérimentations de nouvelles semences ou de nouveaux engrais, telles sont les principales actions techniques réalisées de bout en bout par l'expert et son équipe. Secondé par son équipe, l'expert va procéder aux relevés, aux prises de cotes précises, 
aux calculs de résistance, autant de procédures préalables au démarrage du chantier. Les matériaux recueillis sont ensuite regroupés et les équipes de travail mises en place. L'expert reviendra plusieurs fois par semaine pour contrôler la bonne exécution du travail, pour résoudre un problème technique, pour gérer un conflit entre les membres d'une communauté rurale ou encore pour apaiser des communautés voisines inquiètes de voir un cours d'eau dévié ou tout simplement modifié. Aux moments clefs des chantiers, comme par exemple, la pose d'une chape de béton, l'expert n'hésitera pas à travailler avec les hommes et les femmes des communautés pendant toute une nuit pour "donner l'exemple".

Non content de construire des ouvrages techniques, l'expert se fait dans le même temps pédagogue et intervient à tout moment pour résoudre les tâtonnements, les hésitations et pour relancer la dynamique de l'intervention. Porteur d'une conception instrumentale du changement social, l'expert doit utiliser des techniques dans l'esprit même du Programme pour éviter toute "mauvaise appréciation par les paysans des possibilités offertes par les actions du Programme qui conduirait à une attitude critique négative à l'égard du Programme" ${ }^{28}$. Une grande partie du travail des experts revient donc à gérer les attitudes des communautés. Animateur, il doit motiver les paysans, garantir "la participation des représentants et de la population pour la réalisation des ouvrages et faciliter la coordination entre la réalisation des projets et la contrepartie de la main-d' œuvre formée par la population" ${ }^{29}$. Il oriente et "éduque" les attitudes des communautés concernées par le Programme. Agir dans le sens d'un type de relation sociale se traduit également par l'organisation des lieux de diffusion de formation et de regroupements des individus. Le Programme construit des centres de formation, des salles de réunions et organise des cours de vulgarisation agricole. L'expert y transmet différentes techniques de production, de gestion d'exploitations et de commercialisation. "Participation, collaboration, coopération" sont les principaux principes qui guident son intervention.

Souvent contraint de prouver à posteriori l'utilité d'une technique importée par le Programme sur le terrain, l'expert et les ingénieurs boliviens s'emploieront, par exemple, pendant plusieurs heures à labourer un petit lopin rocailleux pour convaincre les membres de la communauté assis sur un muret, observateurs et étrangement muets, de la pertinence de la technique proposée. Deux logiques différentes se confrontent. Pour les experts, la pertinence de la charrue est évidente et son acceptation par les agriculteurs doit entrainer tout naturellement l'acceptation du Programme européen. Pour les agriculteurs de la communauté au contraire, l'acceptation d'une technique quelle qu'en soient les avantages, ne peut être qu'une conséquence de l'acceptation préalable du principe d'intervention du Programme sur les terres communautaires. Le principe politique prime sur le principe technique.

41 Propagandiste et publicitaire ; l'expert fait, enfin, du prosélytisme européen malgré lui. L'expert passe une grande partie de son temps à faire connaître le projet de développement. L'observation montre qu'une prise de contact, aussi infime soit elle, avec le Programme entraîne, à plus ou moins long terme, la réalisation d'une action technique auprès de toutes les communautés avec lesquelles il est entré officiellement en contact.

Des "bricoleurs"

42 Constamment pris en tenaille entre des intérêts différents, les experts réalisent finalement un véritable travail de "bricolage" ${ }^{30}$. Plus que de simples exécutants des 
volontés techniques de l'institution commanditaire, ils réalisent un immense travail social. Fondamentalement non scientifiques, les experts mènent de front un grand nombre de tâches diversifiées sans subordonner "chacune d'elles à l'obtention de matières premières et d'outils, conçus et procurés à la mesure de son projet" ${ }^{31}$. L'opportunité, l'aléa, telle ou telle rencontre sont fréquemment des facteurs qui entrent en ligne de compte et déterminent une intervention en faveur d'une communauté plutôt qu'une autre.

Parce qu'il est "bricoleur", l'expert improvise. Il fait face aux situations qui se présentent à lui quotidiennement sur l'Altiplano en fonction de ce qu'il a déjà vu et vécu et non en fonction d'une théorie et d'un corps de concepts qui lui permettraient peut-être de dépasser cette expérience héritée. Sans préparation préalable, il compose sur le champ des solutions. Collectionneur d'expériences pendant sa vie professionnelle, l'expert est plus à l'aise avec des situations semblables à celles déjà vécues. Par un travail d'analogie et de rapprochement, il va mettre au service des communautés toute son ingéniosité.

Plus que d'autres, l'expert est sensible aux signes et à "une poésie du bricolage" 32 : sensibilité à ses propres signes d'abord (une plaque commémorative dans laquelle il symbolise son travail accompli, même si son nom n'y figure pas), mais aussi sensibilité aux signes des communautés rurales. Les cérémonies d'inauguration traduisent un début de rencontre et là encore, le rôle de l'expert vient ajouter un supplément d'âme au Programme. Ce dernier fait valoir une relation mécaniste entre le monde technique et le monde social, dont l'expert est, rappelons-le, dans un premier temps, partie prenante, et oublie la fonction essentielle jouée par les systèmes symboliques qui permettent réellement un passage entre ces deux mondes. Aucune condition préalable n'est véritablement mise en place pour que l'expert accède à cette sphère symbolique des communautés andines. Or, il semble bien, qu'à travers ces fêtes, tout se passe comme si l'expert "sentait" toute l'importance de ces rituels pour les membres des communautés. Sans jamais expliquer ou encore moins systématiser cette impression, l'expert s'arrange toujours pour passer ne serait-ce qu'un instant "afin de ne pas froisser les communautés" ${ }^{33}$. Il s'attachera, sans cesse, à convaincre les fonctionnaires de Bruxelles qui passent en coup de vent sur le terrain à ne pas se dérober à ces cérémonies qui "ne sont pas du temps perdu".

"Bricoleur", l'expert est, enfin plus que tout autre acteur du cycle institutionnel de l'aide et tout particulièrement des décideurs de Bruxelles, capable de vivre sur l'Altiplano. Car il sait ce qu'est la vie sur le terrain, le travail avec les agriculteurs ou la vie quotidienne dans des régions hostiles par leur extrême altitude (les Andes), ou par une extrême aridité (le Sahel). Le danger de mort est probablement là plus qu'ailleurs, quasi palpable. L'expert vit dans des zones éloignées des centres de soins, il circule à longueur de journées sur des pistes périlleuses et il est confronté à plusieurs reprises à des situations sociales parfois explosives. Tous ces dangers vécus au quotidien font des experts, des hommes "bien trempés".

Bref, aux prises avec une multitude d'intérêts et d'enjeux contradictoires, l'expert s'emploie à consolider un consensus extrêmement fragile et à tout moment susceptible d'être mis à mal. Médiateur permanent entre l'institution commanditaire du Programme et les réalités boliviennes, l'expert invente les termes d'une action localisée d'aide, définit un espace, repère les attributaires, traduit leurs demandes en besoins, établit un contact précaire entre les communautés et le Programme. Il constitue le lien 
entre les volontés politiques de coopération européenne dans le monde et les réalisations des actions de développement auprès des communautés rurales. Porteur de normes, l'expert diffuse auprès de ces communautés, sanction à l'appui (pouvoir de sélection), des normes techniques et sociales que des pouvoirs politiques ont décidées. En même temps, il se fait l'écho, même s'il les déforme, de pratiques et de savoir-faire locaux et contribue à mettre en forme des normes expérimentées par les acteurs locaux, attributaires de l'aide.

L'analyse de la mise en actes de l'expertise sur les différentes phases d'un projet de développement de la coopération européenne en Bolivie révèle toute l'ambiguïté de cette nouvelle profession aux contours encore bien imprécis. Jugée sur son aptitude à effectuer certains actes et à résoudre des problèmes dans des situations déterminées, l'expertise est avant tout une procédure d'actions. Ce faisant, elle est sans cesse confrontée au problème de la reconnaissance de ses actions et à travers elles d'une reconnaissance d'une forme de compétence. Elle remet ainsi à l'ordre du jour toute la question de l'intelligibilité de l'activité pratique ${ }^{34}$. Pour cela, l'expertise opère une véritable manipulation. En même temps qu'elle s'attache à mettre en œuvre un certain nombre de connaissances, fondées sur l'expérience, sur le terrain, l'expertise travaille sans relâche sur les jugements portés sur elle. Ce double jeu conforte le caractère insaisissable et illusionniste de l'expertise. Elle joue, en quelque sorte, tout à la fois, le rôle de metteur en scène qui réalise, et le rôle de critique qui valide et juge les actions mises en scène. Patient travail de "bricolage", reconnaissable par une manière d'agir qui ne se réduit pas l'application d'éventuelles règles formalisées, l'activité d'expertise tient de l'art, dans la mesure où elle tend à être défini par ce qui est fait et mis en actes.

\section{NOTES}

1.M. Weber, Essais on sociology, trad. anglaise, Oxford University press, 1946, p. 678.

2.A. le Naëlou, Les effets des politiques de développement à l'égard des pays du Tiers monde sur la construction d'une identité de la Communauté Économique Européenne (1960-1990), Thèse de Doctorat de l'EHESS, Sociologie, Paris, 1991, 524 p.

3.A. Le Naëlou, Op. cit., Chap. VII "Sémiologie d'un projet".

4.J. Berque, J. P. Charnay, Pour une sociologie de l'assistance technique. De l'impérialisme et de la décolonisation, Paris, Ed de Minuit, 1965, pp. 433-444.

5.L'étude de la naissance de la charge d'experts montre que la diversité des mesures et le passage à un système de mesures modernes et rationnelles ont entraîné des conflits que les expertises étaient chargées de résoudre. Cf. Les mesureurs du XVIIIème siècle, ancienne forme d'expertise, étudiée par P. Fritsch in Situations d'expertises et experts systèmes, Situations d'expertises et socialisations des savoirs, Actes du C.R.E.S.A.L., Saint Étienne, 1985, pp. 15-48.

6.P. Roqueplo, Le partage du savoir. Science, culture et vulgarisation, Ed du Seuil, Paris, 1974, $254 \mathrm{p}$.

7.G. Balandier, Le détour. Pouvoir et modernité, Paris, Fayard, pp. 124-126. 
8.P. PH. Druet, P. Kemp, G. Thill, Le rôle social de l'expert et de l'expertise, Esprit, 1980, 10, pp. 55-67.

9.M. de Bonis, Les théories implicites de la personnalité en psychiatrie, étude de l'énoncé d'attribution de traits dans l'expertise psychiatrique de 1967 à 1976, Université Paris V, Thèse de doctorat, 1982 ; Langage naturel et expertise psychiatrique. Les marques de quantités dans la description des sujets expertisés : précision ou exactitude ?, Droit et Société, mai 1986, pp. 251-261.

10.J.Y. Caro, Scientificité et rapports sociaux, Sociologie du travail, $\mathrm{n}^{\circ} 1,1984, \mathrm{p} .20$.

11.P. Fritsch, Situations d'expertises et socialisation des savoirs, op. cit. 407 p.

12.F. Dreyfus, La professionnalisation dans l'administration. Recherches sur la fonction idéologique de l'expertise, Annuaire international de la fonction publique, 1975-1976, pp. 295-311.

13.D. Memmi. Savants et maîtres à penser, Actes de la recherche en sciences sociales, $\mathrm{n}^{\circ}$ 76-77, mars 1989, pp. 82-103.

14.P. Bourdieu, Ce que parler veut dire, Paris, Fayard, 1982, p.113.

15.Une fois le principe d'attribution d'une aide à la Bolivie décidé en 1983, la Commission charge officiellement un bureau d'expertise européen d'identifier des thèmes d'actions. Une première mission élabore un constat de la situation nationale (février et mars 1983). Chargée de rechercher un compromis entre les objectifs du plan d'urgence bolivien et les directives de l'aide européenne, l'expertise insiste sur le caractère délité de la société bolivienne. La situation du département est évaluée, afin de dégager une hiérarchisation des besoins urgents pour une intervention rapide. De ce premier constat, peu d'éléments sont retenus par la Commission. Elle retiendra toutefois l'idée d'une collaboration avec les Corporations Régionales de Développement des départements d'Oruro et de Potosi. Deux nouvelles missions d'expertise sont alors renvoyées (mai-juin 1983) dans l'un et l'autre de ces départements au terme desquelles deux rapports de mission sont remis juin 1983) à la Commission. Ces seconds rapports rendent compte des activités des Corporations Régionales de Développement de Potosi et d'Oruro, donnent une appréciation des projets proposés par les services techniques boliviens, proposent des zones et des modalités d'application de l'aide, des possibilités de développement ultérieur de celle-ci et définissent le rôle d'une assistance technique européenne.

16.Le terme d"Europe" désigne dans ce texte la Communauté des douze États européens et non l'Europe toute entière.

17.0. Piron, Décision et monde rural, Études rurales, n¹01-102, janvier-juin 1986, pp. 61-72.

18."La Communauté Européenne place, auprès des Corporations Régionales de Développement, une équipe réduite d'assistance technique, pour un appui spécifique à l'exécution du Programme et pour un temps limité au maximum à la durée de son exécution. Cette équipe, placée sous la responsabilité du président de la Corporation, doit compléter l'identification des micro-projets, établir le programme des réalisations et veiller à leur bonne exécution, assurer la formation de techniciens et de formateurs et effectuer les études à leur portée et qui s'avéreraient nécessaires, gérer, en liaison avec les Corporations, les fonds communautaires affectés au Programme et ceux destinés au crédit agricole" Convention de financement $n^{\circ} \mathrm{NA} / 83 / 7, \mathrm{p} 14$. 19.Les experts européens arrivent en Bolivie en décembre 1983. En atterrissant à l'aéroport "Del Alto" de La paz, ils sont passés en l'espace de quelques heures du niveau de la mer à une altitude de $4000 \mathrm{~m}$. C'est là un avant-goût de la véritable mise à 
l'épreuve qui les attend. Ils doivent surmonter le "choc" de l'arrivée, supporter le mal d'altitude ou déjà le prévenir en buvant une boisson qui leur est inconnue (tisane à base de coca). Ils ne connaissent pas la région dans laquelle ils vont vivre, ils ne connaissent pas les communautés rurales avec lesquelles ils sont appelés à travailler, et une grande part d'entre eux parlent encore mal un espagnol récemment acquis dans un cours de formation accéléré avant de partir. Rapidement, en professionnel, les experts doivent "donner le meilleur d'eux-mêmes", montrer "ce qu'ils ont dans le ventre", "faire face" à une situation hors du commun. Car vivre et travailler dans la région de l'Altiplano est une situation qui sort de l'ordinaire pour un Européen. Relever ce défi dans une situation d'incertitude de surcroît, imprègne leur existence quotidienne.

20.Sofipro Belgium, Étude d'un programme de Micro projets d'Investissements Ruraux dans le département de Potosi, mai juin 1986, 84 p + annexes, pp. 13-20.

21.SOFIPRO Belgium, op. cit., $\mathrm{p} 16$.

22.La notion de territoire entendue ici indique un pouvoir qui se construit un espace d'action.

23.Il s'agit d'un centre expérimental sur les races ovines (El Chorro), d'un centre expérimental sur les races andines de camélidés (Turco), d'un centre expérimental de forestation pépinières et d'élevages de lapins et de chinchillas (Cadea) et d'une zone expérimentale de quinoa (Salinas).

24.Il s'agit de la "Corporation" régionale, de "l'Institut des technologies agricoles", de la "S.E.N.A.C.", de la "Federacion Unica de los Trabajadores Desocupados" et enfin des autorités et des représentants des cantons et communautés de Romos, Otavi, Puna, Mobona dans la province de Linares, Olliuchi, Llallagua, Uncia et Aymaya dans la province de Chayanta et enfin Calacala, Pocoata, et Estancias dans la province de Bustillos.

25.Il s'agit ici non plus de la phase d'identification du Programme, mais de l'identification des micro-projets différée au stade de l'exécution.

26.Cf. P Bourdieu, L Boltanski, Le titre et le poste, Actes de la recherche en sciences sociales, (2), 1975, pp. 95-107.

27.M. Marie, Un territoire sans nom, pour une approche des sociétés locales, op. cit. p 54.

28.SOFIPRO Belgium op. cit., pp. 16-18.

29.Idem, p. 3.

30.C. Levi Strauss, La pensée sauvage, Paris, Plon, 1962, pp. 26-47.

31.Idem, p 27.

32.Idem, $\mathrm{p} 32$.

33.Les experts ont des rôles à remplir qui rappellent ceux des "cadres" étudiés par $\mathrm{L}$. Boltanski, Les cadres, Paris, Ed de Minuit. 1982, pp. 47-51, (voir par exemple, leur interchangeabilité théorique). Mais ils ont également une sensibilité qu'ils forgent par leur contact permanent avec le terrain. Cet humanisme pragmatique les distingue alors des "cadres" de Bruxelles.

34.C'est sur ce point qu'intervient tout le débat sur la possibilité et les difficultés à réduire le cours d'action à la réalisation d'un ou plusieurs algorithmes ou systèmes-experts. Cf. par exemple, J. Merchiers, P. Pharo, Éléments pour un modèle sociologique de la compétence d'expert, Sociologie du travail, $\mathrm{n}^{\circ}$ 1, 1992, pp. 47-63 et M. de Fornel, Connaissances procédurales et déclarative dans l'interaction médicale, Qu'est ce qu'un expert?, Réseaux, nº 41, CNFT, 1990. 